\title{
EVALUACIÓN DEL CONOCIMIENTO INDIGENA KICHWA COMO HERRAMIENTA DE MONITOREO EN LA ABUNDANCIA DE ANIMALES DE CAZA
}

\author{
Pedro E. PÉREZ-PEÑA ${ }^{1,2,}$, Lourdes RUCK ${ }^{1}$, María S. RIVEROS $^{3}$, Gloria ROJAS $^{1}$ \\ 1 Servicio Nacional de Áreas Naturales Protegidas por el Estado (SERNANP), Reserva Nacional Pucacuro. \\ pedperpe@hotmail.com \\ 2 Yavarí: Conservación y Uso Sostenible (YAVACUS) \\ 3 Servicio Nacional de Áreas Naturales Protegidas por el Estado (SERNANP), Reserva Nacional Matsés
}

\section{RESUMEN}

Se evaluó la percepción indígena kichwa sobre la abundancia de animales de caza usando la teoría de consenso cultural. Se calculó la densidad y biomasa obtenida con el método de transectos lineales y en banda. Se entrevistó a 25 cazadores kichwas y se recorrió 12 transectos lineales sumando un esfuerzo de $556.1 \mathrm{~km}$ en la zona de caza de la Reserva Nacional Pucacuro. La percepción de los cazadores sobre la abundancia de animales de caza estuvo significativamente correlacionada con la densidad y biomasa, sin embargo, la abundancia percibida de Dasyprocta fuliginosa, Nasua nasua y Tapirus terrestris fue diferente a los resultados de censos debido a la subestimación de los censos por transectos. Se propone una ecuación de regresión exponencial para calcular la densidad de animales de caza en base a scores de percepción de abundancia de cazadores. El conocimiento local de los kichwas debe ser tomado en cuenta en los programas de monitoreos de animales de caza.

PALABRAS CLAVES: Kichwas, Consenso Cultural, Distance, Abundancia, Animales de caza

\section{ASSESSING KICHWA INDIGENOUS KNOWLEDGE AS A MONITORING TOOL FOR GAME ABUNDANCE}

\begin{abstract}
The Kichwa peoples' perception of game abundance using cultural consensus was assed and compared with density and biomass calculated from line and width strip transects. Twenty-five hunters were interviewed and twelve transects were walked multiple times resulting in $556.1 \mathrm{~km}$ of sampling effort. The Kichwa peoples' perception of game abundance was significantly correlated with density and biomass, however the perceived abundance of agouti Dasyprocta fuliginosa, coati Nasua nasua and tapir Tapirus terrestris differed from census results due to underestimation in line transects. We propose an exponential regression equation to calculate game density from hunter's perception. The local knowledge of the Kichwa people could be usefully incorporated into game monitoring programs.
\end{abstract}

KEYWORDS: Kichwas, Cultural consensus, Distance sampling, Game abundance 


\section{INTRODUCCIÓN}

El monitoreo de los recursos naturales es fundamental para evaluar el éxito de las acciones de conservación dentro o fuera de áreas protegidas. Los monitoreos, que son logísticamente muy costoso, deben ser bien diseñados y planificados para que las fluctuaciones o tendencias observadas sean de las poblaciones de animales en estudio y no de las diferencias en tiempo, espacio o capacidad del personal (Elzinga et al., 2002). Debido a la limitación del presupuesto asignado, muchos proyectos de conservación realizan evaluaciones solo en un momento dado y no a través del tiempo, por lo tanto la búsqueda de buenas estrategias y diseños de bajo costo son esenciales para el desarrollo de un plan de monitoreo, mas aún si están enfocadas al monitoreo de recursos explotados tradicionalmente, como los animales de caza (Rowcliffe, 2002).

Los animales de caza son la fuente de proteínas e ingresos económicos del poblador amazónico (Bodmer et al., 2004). Ancestralmente, la caza de las comunidades indígenas era sostenible porque sólo era para autoconsumo, pero al pasar el tiempo, el incremento demográfico y mayor demanda de recursos ocasionaron la disminución y en algunos casos la desaparición de las especies de carne de monte, debido a la susceptibilidad a la sobrecaza (Bodmer et al., 1997) además de otros factores como enfermedades (Fragoso, 2004), escasez de alimento (Haugaasen \& Peres, 2008), baja productividad reproductiva (Hurtado-Gonzales \& Bodmer, 2004) o cambios en el sex ratio al nacimiento (Pérez-Peña, 2007; Fang et al., 2008). En este sentido, los animales de caza, quienes brindan bienestar a grandes sectores de la sociedad, no deben de estar ausentes en las agendas de conservación y deben de ser monitoreadas a largo plazo para tomar las medidas correctivas en el momento oportuno.

En varios lugares de la Amazonia peruana, los animales de caza se monitorearon mediante el uso de transectos lineales (Pérez-Peña, 2007; Fang et al., 2008; Bodmer et al., 2011), y aplicando el método Distance (Buckland et al., 1993), sin embargo, estos programas de monitoreos necesitan grandes presupuestos y esfuerzo de muestreo, lo que resulta en una actividad impensable y desmotivadora para muchos proyectos de conservación. El monitoreo necesita usar herramientas metodológicas adecuadas, comparables en muchas regiones, de bajo costo y fácil de implementar dentro o fuera de áreas protegidas.

Las entrevistas a través de la teoría de consenso cultural (Weller, 2007) puede ser una gran alternativa para monitorear los animales de caza, porque es de bajo costo, fácil de implementar y se basa en el conocimiento tradicional de los pobladores locales sobre los recursos naturales. El conocimiento de los recursos naturales por lo pobladores locales no esta en duda; los indígenas Guarayos de la Amazonía boliviana y los Matsés de la Amazonía peruana son grandes conocedores de la ecología y abundancia de los animales de caza (Van Holt et al., 2010; Voss \& Fleck, 2011), los indígenas Cashinahuas tienen amplio conocimiento metódico en abundancias de plantas muy similar al conocimiento académico (Pitman et al., 2011). Esto es sólo una pincelada del conocimiento indígena sobre la abundancia de los recursos naturales lo cual puede ser usado como instrumento de monitoreo mediante el uso del consenso cultural.

Los indígenas Kichwas de la comunidad de 28 de Julio vivieron ancestralmente en las cabeceras del río Pucacuro y descendieron hasta llegar al rio Tigre donde se establecieron cerca al pueblo de Intuto, capital del distrito del Tigre, Provincia de Loreto. La cacería de los Kichwas en la cuenca del río Pucacuro, se realizó desde el siglo pasado y creó un amplio conocimiento de las poblaciones de animales de caza al interior de esta cuenca, sin embargo es necesario traducir este conocimiento local al conocimiento académico o científico y evaluar su similitud.

El presente estudio evalúa la utilidad de las entrevistas a cazadores indígenas Kichwas a través de la teoría de consenso cultural como un método de evaluación de animales de caza mediante la comparación de densidades resultados obtenidos con transectos lineales en la zona de caza de la Reserva Nacional Pucacuro, al norte de la Amazonía Peruana. Este resultado será muy útil para crear estrategias en el monitoreo de varias especies de caza de una forma práctica, de bajo costo, con apoyo voluntario de cazadores, generando información a largo plazo y una atmosfera favorable para la discusión del manejo sostenible de animales de caza.

\section{MATERIALES Y MÉTODOS}

\section{ÁREA DEESTUDIO}

Los censos de fauna silvestre se realizaron mediante transectos lineales en la zona de caza de la Reserva Nacional Pucacuro. Esta reserva se ubica en el río del mismo nombre, Pucacuro, afluente del río Tigre por su margen izquierda; situada en el Distrito del Tigre, Provincia de Loreto, Departamento de Loreto. La cuenca del río Pucacuro esta en un Bosque húmedo tropical, la temperatura media anual fluctúa entre 25.2-25. ${ }^{\circ} \mathrm{C}$ (estación meteorológica del Curaray). Durante los meses de Junio y Julio, ocurren periodos breves de descenso moderado de la temperatura (que ocasionalmente bajan hasta 14 ó $15^{\circ} \mathrm{C}$ ), provocados por vientos del hemisferio sur ("fríos de San Juan"). 
La precipitación promedio anual fluctúa entre 2300 y $2900 \mathrm{~mm}$. Generalmente entre los meses de Julio y Noviembre, se da la temporada de menor intensidad de lluvias. El nivel del río es mayor entre los meses de Febrero y Agosto (época de creciente), mientras que entre los meses de Agosto y Diciembre el nivel del río es bajo pero con algunos periodos breves de creciente.

Los transectos lineales en la zona se establecieron abarcando diferentes tipos de hábitats: terraza baja, terraza alta, aguajal y bosques de colinas. Los bosques de terraza baja se caracterizaron por presentar inundación temporal, relieve ligeramente plano, donde se pueden encontrar las "restingas" con ñejillales Bactris spp. y otras palmeras como huacrapona Iriartea deltoidea, huasaí Euterpe precatoria, además se encuentran los ya conocidos irapayales Lepidocaryum tenue. Los bosques de terraza alta se caracterizaron por no ser inundables y tener relieve ligeramente plano con algunas depresiones, con formaciones palmáceas dominantes como el hungurahui Oenocarpus batahua, irapay L. tenue y shapaja Attalea racemosa. Los bosques de colina baja no son inundables y mostraron relieves ondulados, con o sin quebradas en las depresiones. Las palmeras dominantes en este tipo de bosque fueron el hungurahui $O$. batahua, irapay $L$. tenue, shapaja A.racemosa y palmiche Geonoma spp. Los pantanos palmáceos o aguajales tuvieron relieve plano de muy mal drenaje e inundados permanentemente. Las palmeras dominantes fueron Mauritia flexuosa, huasaí E. precatoria, cinamillo $O$. mapora y en algunas partes el huicungo Astrocaryum тигитити. Las entrevistas a cazadores Kichwas se realizaron en la comunidad de 28 de Julio. Actualmente, la cacería de los Kichwas al interior de la reserva está restringida solo a la zona de caza, según el mutuo acuerdo entre la jefatura de la reserva y las autoridades Kichwas. De esta forma, los resultados de densidades de transectos y percepción de la abundancia de cazadores fueron obtenidos del mismo lugar: la zona de caza de la reserva, ubicada entre la quebrada Pañayacu y la parte baja de la quebrada Baratillo y Tangarana (Figura 1).

La comunidad de 28 de Julio esta compuesta por 559 habitantes, de éstos, 315 son hombres y 244 son mujeres. Los niños (0-11 años) conforman el 52.4\% de la población total, mientras que los jóvenes (1217 años), adultos (18-59 años) y ancianos ( $>60$ años) conforman el 7.5, 37.0 y $3.0 \%$ respectivamente. Los Kichwas realizan tres actividades principales para mantener a sus familias: la agricultura $(93.7 \%)$, pesca $(84.4 \%)$ y cacería (81.3) pero disfrutan más realizando la agricultura $(58.1 \%)$ y cacería $(51.6 \%)$ y obtienen mayores beneficios económicos con la cacería (59.4) y agricultura $(50 \%)$.

\section{COLECTADEDATOS}

\section{Censos por transectos lineales}

Se aperturaron 12 transectos lineales en la zona de caza de la reserva, distribuidas en tres localidades: Serna, Posayo y Pañayacu (Figura 1). Los transectos en cada zona tenían direcciones de apertura entre 80$90^{\circ}$ y tuvieron diez pseudoréplicas, las cuales sumaron un esfuerzo total de $556.1 \mathrm{~km}$. Se creó cuatro grupos de evaluadores, cada uno conformado por un profesional con amplia experiencia en censos de animales de caza y un cazador Kichwa, cazador (guardaparque), con la finalidad de garantizar la calidad en la colecta de información y mayor cantidad de avistamientos.

Los censos de animales de caza fueron realizados mediante el método Distance en transectos lineales (Buckland et al., 1993), que consistió en registrar la distancia perpendicular del animal avistado a la trocha. Si la especie fue gregaria se calculó la distancia perpendicular del primer individuo avistado al transecto. Los transectos tuvieron entre 3.5 y $5 \mathrm{~km}$ de longitud y fueron recorridos entre las 7:00 y 15:00 horas, registrando las diferentes especies de mamíferos y aves de caza. De forma paralela se colectó información ecológica como tipo de hábitat y formaciones de palmeras dominantes. Otras informaciones colectadas fueron: fecha, lugar, número de individuos, ubicación del transecto, hora, distancia recorrida, clima y comportamiento del individuo (sólo si éste continuaba con sus actividades en forma natural).

La técnica está basada en el principio de que los evaluadores no pueden observar todos los animales que se encuentran fuera de la línea central del transecto y la probabilidad de avistamiento de un animal depende de la distancia del animal al centro de la línea. Los animales más cercanos al transecto tienen mayor probabilidad de ser observados que los animales más alejados. El método Distance estima los animales que pueden ser desapercibidos y los incluye en el estimado de densidad. El método asume que todos los animales que están en el centro de la línea de transecto ( $0 \mathrm{~m}$ distancia perpendicular) son observados y las medidas perpendiculares de los animales a la línea del transecto son tomadas correctamente.

Anchura fija o transecto en banda: este método asume que todos los individuos son observados dentro una distancia predeterminada (ancho fijo) desde la línea central con una probabilidad de 100\% (Burnham et al., 1980). El punto crítico de este método es conocer la distancia de anchura fija o distancia efectiva, que puede variar en función de las especies y criterios de los autores. Si la anchura del transecto es muy amplia no podrá detectar todos los animales dentro del transecto y así los resultados 
estarían subestimados, pero si la anchura del transecto es muy angosta hubiera una muestra muy pequeña con un gran esfuerzo y los estimados serían menos precisos (Meredith, 2008). En este estudio consideramos que la anchura fija fue aquella distancia donde las especies pudieron ser identificadas y contadas apropiadamente dependiendo del tamaño de la especie, tamaño de grupo y comportamiento críptico, y ésta distancia es similar a la anchura efectiva del método Distance en estudios de Salovaara et al., (2003), Pérez-Peña (2007), Fang et al., (2008) y Bodmer et al. (2011).

\section{Entrevistas}

Se entrevistaron a 25 cazadores de la comunidad indígena Kichwa para la evaluación de la percepción de abundancia de los animales de caza utilizando la metodología de Van Holt et al. (2010), mediante figuras laminadas de 23 especies que los entrevistados fácilmente identificaron sin ayuda del entrevistador. Estas imágenes fueron obtenidas de Emmons (1997) y Eisenberg \& Redford (1999) para mamíferos y Schulenberg et al. (2010) para aves. Antes de iniciar las entrevistas, este método fue previamente probado con guardaparques Kichwas de la Reserva Nacional Pucacuro para confirmar la facilidad de identificación de cada una de las especies. La entrevista consistió en presentar las imágenes laminadas a los cazadores y preguntar cuales de aquellos eran animales raros y luego cuales eran abundantes en la zona de caza de la Reserva Nacional Pucacuro, las imágenes que no fueron seleccionados se consideraron como frecuentes. Cuando los entrevistados seleccionaban muchas especies abundantes o raras se volvía a preguntar para ver si coincidían o estaban seguros en sus respuestas, en algunos casos no elegían los mismos animales y dejaban algunas especies fuera del grupo. Este método fue muy útil para evitar confusión con los nombres locales en español o Kichwas porque algunos cazadores hablan menos español; además fue muy ameno porque las imágenes individuales (en forma de naipe) a colores atrae fácilmente sus atención, elimina la timidez y crea un ambiente de confianza para conversar diversos temas de los diferentes animales.

\section{ANÁLISIS DEDATOS}

\section{Transectos lineales}

Los datos fueron analizados con el software Distance 6.0 (Thomas et al., 2009), que permite calcular la densidad individual o grupal y teóricamente necesita un mínimo de 40 avistamientos, sin embargo se pueden obtener resultados confiables hasta con 20 avistamientos, si las distancias perpendiculares están muy bien distribuidas desde la línea central del transecto hacia el punto más lejano (Peres \& Cunha, 2011). La información de transectos lineales fue modelada con combinaciones de tres funciones claves y tres series de expansión recomendadas por Buckland et al. (1993) y Meredith (2008). Los resultados fueron validados por medio del Criterio de Información de Akaike (AIC) que debe de ser el menor, la prueba de bondad y ajuste o Goodness of Fit con un $P>0.05$, y el coeficiente de variación $(\mathrm{CV})<30 \%$. Si los resultados no cumplieron con estos requisitos del software Distance se optó por utilizar el método de anchura fija o transecto en banda.

\section{Anchura fija o transectos en banda}

En este análisis es necesario el número de individuos avistados dentro de la distancia o anchura establecida y el recorrido total. La fórmula para calcular la densidad es: $D=N / 2 W L$ (Burnham et al., 1980, Buckland et al., 1993), donde $D$ es la densidad, $N$ es el número de individuos avistados dentro del ancho establecido, 2 es la constante que indica muestreos a ambos lados del transecto, $W$ es la distancia establecida como ancho fijo (en km) y $L$ la longitud recorrida $(\mathrm{km})$.

\section{Teoría de consenso cultural (tcc)}

Se basa en la asunción que la cultura es un conocimiento compartido, las personas que conocen la respuesta a una pregunta tienden a estar de acuerdo con otros, mientras que las personas que no conocen la respuesta, tratan de adivinar y tienen menos probabilidad en estar de acuerdo con los demás (Weller, 2007). Sin embargo, el consenso de informantes puede ser considerado como el factor más importante para el conocimiento solo si existen las siguientes consideraciones: a) cada informante brinda respuestas independientemente de otros, es decir, no son válidas para entrevistas grupales o consultas a otras personas, b) las preguntas deben de ser sobre un solo tema y con el mismo nivel de dificultad y c) las preguntas deben de tener un único conjunto de respuestas. Este análisis no crea consenso, solo evalúa el nivel de acuerdo o consenso presente.

En este estudio se construyó una matriz de respuesta de cada cazador y se afirmó consenso cultural si la proporción el primer y segundo eigenvalue o autovalor fue mayor a tres (significa que el primer factor explica dos tercios o más de la varianza en la matriz de respuestas). Este modelo proporcionó además los valores de abundancia como los valores promedios de respuesta de los informantes. Estos análisis se realizaron con el programa UCINET 6.45 (Borgatti et al., 2002). 
Comparación entre métodos de transectos y entrevistas

Se realizaron análisis de correlación (datos normalizados con Ln) y regresión exponencial de los scores de abundancias (variable independiente) y densidad poblacional con Bioestat 5.0 (Ayres et al., 2007). Para comparar los valores de densidad y scores de la percepción de cazadores, previamente se transformaron a categorías de clases de abundancias. La amplitud del score se dividió entre el número de clases para generar los límites de cada categorías, mientras que en el caso de las densidades se utilizó fuentes bibliográficas mostrando valores de densidad de lugares con ligera o ninguna alteración como referencia de valor de densidad más alta (capacidad de carga), a partir del cual se infirió valores en categoría: abundante, frecuente y raro.

Se tomó este criterio porque tiene más sentido biológico; de lo contrario Nasua nasua y Tapirus terrestris estuvieran en la misma categoría por tener igual densidad $\left(0.14 \mathrm{ind} / \mathrm{km}^{2}\right)$, sin embargo, los límites de capacidad de carga de éstas especies son diferentes, la primera especie puede llegar hasta 15 ind. $/ \mathrm{km}^{2}$ mientras que la segunda llega hasta 0.8 ind. $/ \mathrm{km}^{2}$, por lo tanto el valor de densidad de 0.14 ind. $/ \mathrm{km}^{2}$ no puede estar en la misma categoría de abundancia de acuerdo al sentido biológico.

\section{RESULTADOS}

La población cazadora en la comunidad kichwa de 28 de Julio tiene una edad promedio de $38.6 \pm 12.4$ (SD) pero el $50 \%$ de la población $(25-75 \%$ intercuartiles) tiene una edad entre 28.5 - 42.7 años con un rango entre 22 y 70 años. Los kichwas comienzan a cazar a una edad promedio de $17.6 \pm 4.8$ años, el 50\% de los cazadores comienzan a cazar entre los 14.2 y 20 años. La cacería diurna en la reserva en promedio es de $3.9 \pm 2.2$ horas y la nocturna $3.3 \pm 1.87$. Los cazadores manifestaron que los lugares donde encuentran más animales de caza son las colpas $(62.5 \%)$, orilla de río $(40.62 \%)$ y aguajales en tiempo de fructificación (18,8\%).

Los cazadores compartieron la percepción de la abundancia de animales de caza, reflejada en el valor de la proporción del primer y segundo eigenvalue mayor a tres $(12.683 / 1.674=7.575)$. Los cazadores coincidieron en que las especies más abundantes en la reserva fueron la huangana Tayassu pecari, el choro Lagothrix poeppigii, fraile Saimiri sciureus, majas Cuniculus paca, sajino Pecari tajacu y pucacunga Penelope jacquacu y las especies más raras fueron el puma Puma concolor y el huron Galictis vittata (Tabla 1). Los censos por transectos lineales analizados con el método Distance y anchura fija mostraron como especies más densas al mono choro Lagothrix poeppigii, fraile Samiri sciureus, pucacunga Penelope jacquacu y huangana Tayassu pecari y a los menos densos al shihui Tamandua tetradactyla, venado rojo Mazama americana y sachavaca Tapirus terrestris (Tabla 2).

La percepción de abundancia y las densidades de las especies de caza mostraron alta correlación significativa, (Figura 2), es decir, los patrones de las curvas de orden de especies y densidad son similares entre ambos métodos.

De acuerdo a las categorías establecidas, ocho de las once especies coinciden en la misma clase de abundancia usando ambos métodos; estas especies son el mono choro L. poeppigii, fraile $S$. sciureus, pucacunga $P$, jacquacu, huangana $T$. pecari, venado cenizo Mazama nemorivaga, venado rojo Mazama americana y shihui $T$. tetradactyla. No todas estas especies presentan densidades altas, algunas son raras de encontrar (Tabla 3).

Los resultados de ambos métodos no están relacionados con el tamaño de la especie en términos de masa $(\mathrm{kg})$ pero si están correlacionados positivamente y significativamente con el tamaño de grupo que lo conforman (Tabla 4).

Si usamos los resultados de entrevistas de las especies coincidentes, podemos predecir sus densidades con un alto nivel de confianza. Un análisis de regresión exponencial muestra que los valores de scores pueden predecir el $80 \%$ de los valores de densidad significativamente $(\mathrm{P}=0.0024)$ mediante la siguiente ecuación: $\mathrm{Y}=\mathrm{a} e^{\mathrm{bX}}$, en donde $Y$ es la densidad de la especie, $a$ es el intercepto igual a $0.0001, e$ (épsilon) es la constante $2.718281, b$ es el coeficiente de regresión 3.7857 y $X$ es el score promedio de la percepción de cazadores de la abundancia de una especie.

Así, al realizar las entrevistas usando el consenso cultural y la ecuación mencionada se puede calcular la densidad poblacional de las especies de caza, pero no solo de las más densas también de las difíciles o raras de encontrar. 


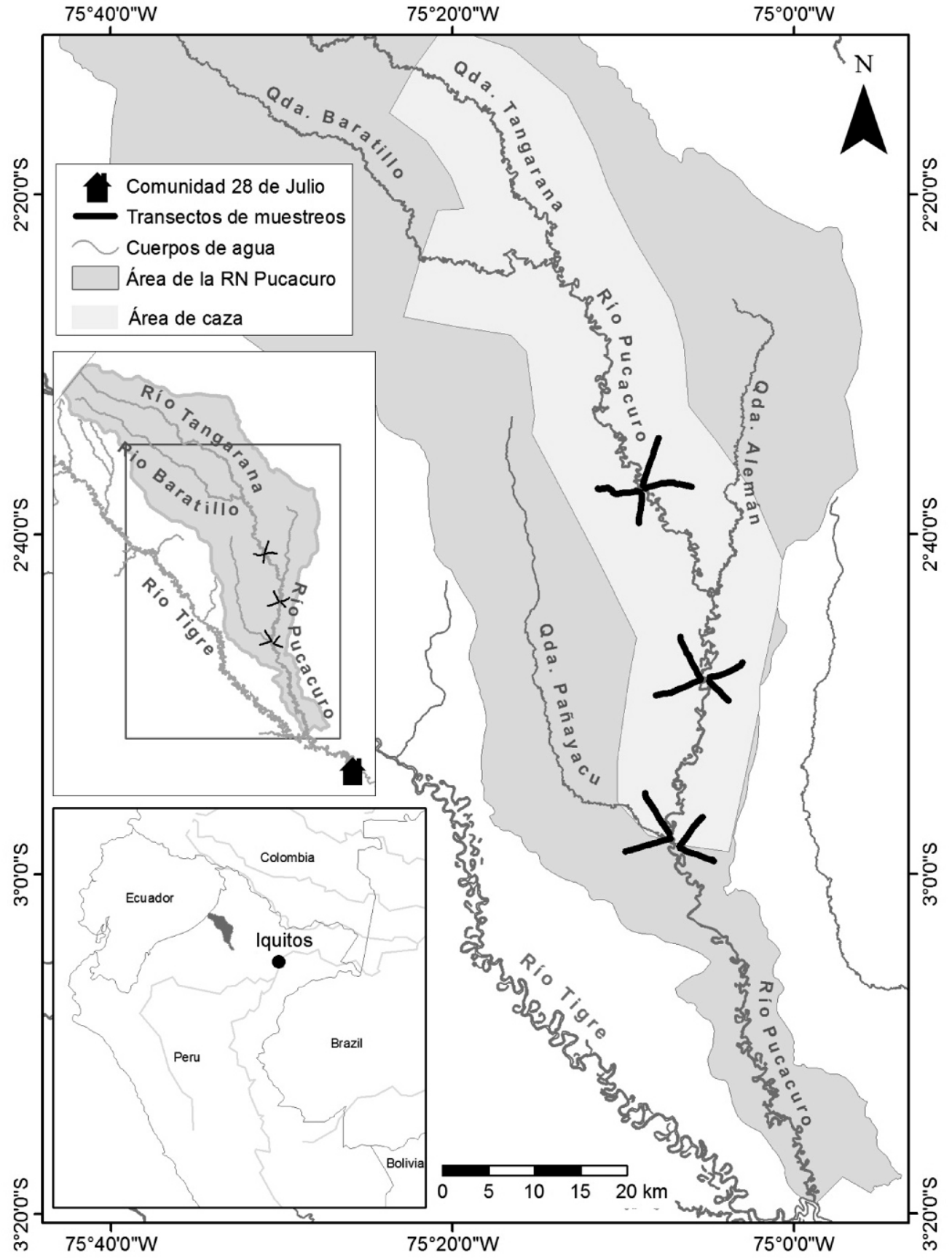

Figura 1. Mapa de la zona de estudio mostrando la ubicación de los transectos lineales, zona de caza y la comunidad Kichwa de 28 de Julio. 


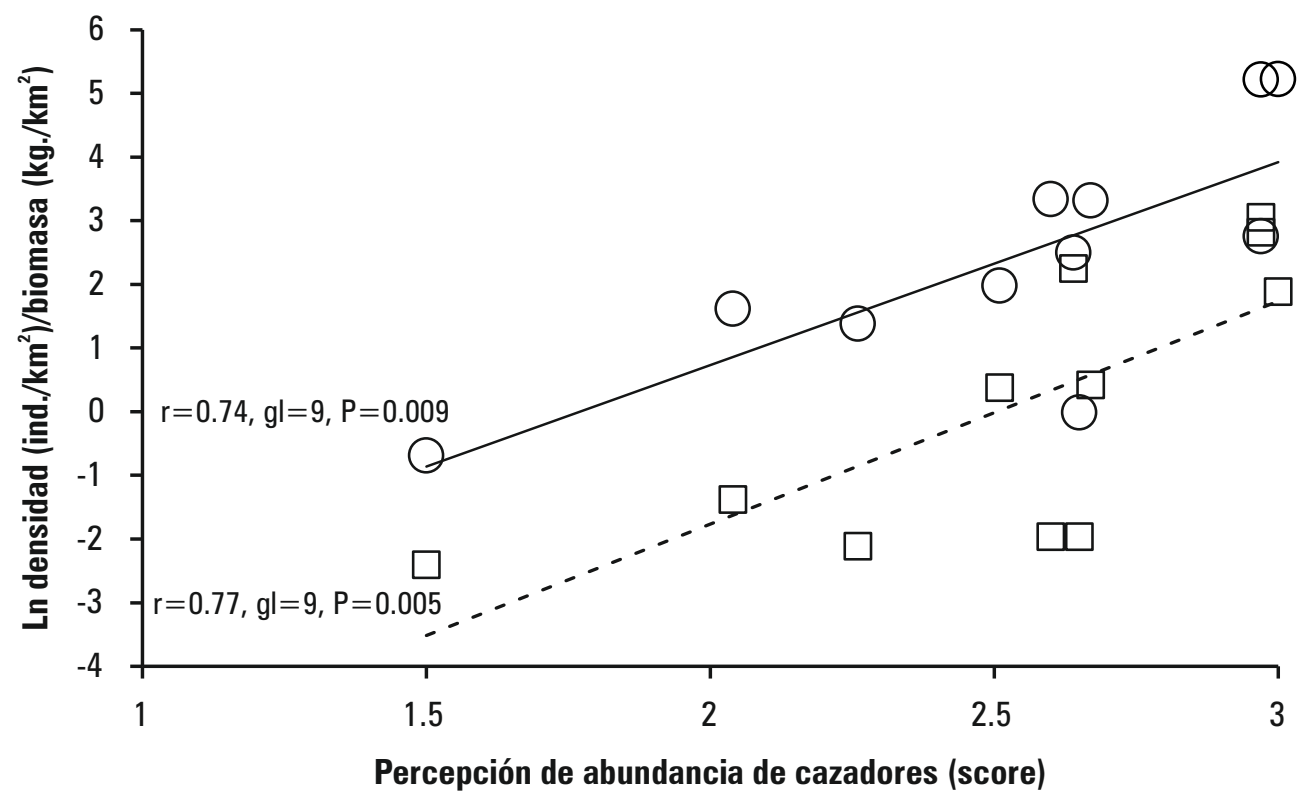
$\square \quad$ score-densidad
o score-biomasa
- - - - Lineal (score-densidad) Lineal (score-biomasa)






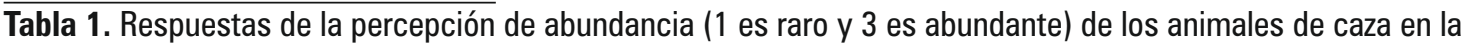
Reserva Nacional Pucacuro $(n=25)$.

\begin{tabular}{|c|c|c|c|c|}
\hline $\begin{array}{c}\text { Nombre } \\
\text { científico }\end{array}$ & $\begin{array}{l}\text { Nombre } \\
\text { común }\end{array}$ & $\begin{array}{l}\text { Nombre en } \\
\text { Kichwa }\end{array}$ & $\begin{array}{c}\text { Peso promedio } \\
\text { (kg) }\end{array}$ & Score \\
\hline \multicolumn{5}{|l|}{ Perisodactyla } \\
\hline Tapirus terrestris & sachavaca & Wagra & $200.00^{1}$ & 2.60 \\
\hline \multicolumn{5}{|l|}{ Artiodactyla } \\
\hline Mazama americana & venado rojo & Puka taruga & $33.00^{1}$ & 2.26 \\
\hline Mazama nemorivaga & venado gris & Ushpa taruga & $20.00^{4}$ & 2.04 \\
\hline Pecari tajacu & sajino & Lumukuchi & $18.00^{1}$ & 2.67 \\
\hline Tayassu pecari & huangana & Gargara & $27.95^{1}$ & 3.00 \\
\hline \multicolumn{5}{|l|}{ Primate } \\
\hline Lagothrix poeppigii & choro & Kushillo & $8.71^{2}$ & 2.97 \\
\hline Saimiri sciureus & fraile & Varisa & $1.01^{2}$ & 2.97 \\
\hline \multicolumn{5}{|l|}{ Rodentia } \\
\hline Coendu bicolor & erizo & Casha Kushillo & $4.00^{4}$ & 1.61 \\
\hline Cuniculus paca & majas & Picuro & $8.00^{1}$ & 2.90 \\
\hline Dasyprocta fuliginosa & añuje & Punllana & $4.96^{1}$ & 2.51 \\
\hline Hydrochoerus hydrochaeris & ronsoco & Kapiwagra & $50.00^{1}$ & 2.67 \\
\hline \multicolumn{5}{|l|}{ Carnivora } \\
\hline Galictis vittata & huron & Sacha aycu & $3.20^{4}$ & 1.25 \\
\hline Leopardus pardalis & tigrillo & Takay puma & $8.00^{1}$ & 1.46 \\
\hline Lontra longicaudis & nutria & Kinti & $9.50^{1}$ & 2.27 \\
\hline Nasua nasua & achuni & Tijun & $7.00^{1}$ & 2.65 \\
\hline Panthera onca & otorongo & Puma & $110.00^{1}$ & 1.44 \\
\hline Pteronura brasiliensis & lobo de rio & Ushpa Lubu & $30.00^{1}$ & 2.40 \\
\hline Puma concolor & puma & Puca puma & $46.00^{1}$ & 1.36 \\
\hline \multicolumn{5}{|l|}{ Edentado } \\
\hline Choloeps sp. & pelejo & Intimalla & $5.40^{4}$ & 1.64 \\
\hline Dasypus sp. & carachupa & Armadillo & $3.50^{1}$ & 2.21 \\
\hline Tamandua tetradactyla & shihui & Añangu kushillu & $5.50^{1}$ & 1.50 \\
\hline \multicolumn{5}{|l|}{ Craciformes } \\
\hline Penelope jacquacu & pucacunga & Karunshi & $1.28^{3}$ & 2.64 \\
\hline
\end{tabular}

'Aquino et al. (2001), ${ }^{2}$ Peres y Dolman (2000), ${ }^{3}$ Terborgh et al. (1990), ${ }^{4}$ Reiss et al., (2006) 
Tabla 2. Densidad de especies de caza en la Reserva Nacional Pucacuro calculados mediante el método Distance y Anchura fija.

\begin{tabular}{|c|c|c|c|c|c|}
\hline Especies & $\begin{array}{c}\text { Distancia } \\
\text { efectiva }(\mathbf{m})\end{array}$ & $\begin{array}{l}\text { Tamaño máximo de } \\
\text { grupo (individuos) }\end{array}$ & $\begin{array}{l}\text { Biomasa } \\
\left(\mathbf{k g} \cdot / \mathbf{k m}^{2}\right.\end{array}$ & $\begin{array}{l}\text { Densidad } \\
\text { (ind./ } / \mathbf{k m}^{2} \text { ) }\end{array}$ & Análisis \\
\hline \multicolumn{6}{|l|}{ Mamíferos } \\
\hline \multicolumn{6}{|l|}{ Perisodactyla } \\
\hline Tapirus terrestris & 20 & 1 & 28 & $0.14 \pm 0.03$ & Anchura fija \\
\hline \multicolumn{6}{|l|}{ Artiodactyla } \\
\hline Tayassu pecari & 30 & 75 & 183.92 & $6.58 \pm 6.44$ & Anchura fija \\
\hline Pecari tajacu & 20 & 8 & 27.36 & $1.52 \pm 0.79$ & Anchura fija \\
\hline Mazama americana & 20 & 1 & 3.96 & $0.12 \pm 0.11$ & Anchura fija \\
\hline Mazama nemorivaga & 20 & 1 & 5 & $0.25 \pm 0.12$ & Anchura fija \\
\hline \multicolumn{6}{|l|}{ Primate } \\
\hline Lagothrix poeppigii & 25 & 25 & 183.39 & $21.05 \pm 4.00$ & Distance \\
\hline Saimiri sciureus & 20 & 48 & 15.64 & $16.60 \pm 6.02$ & Distance \\
\hline \multicolumn{6}{|l|}{ Rodentia } \\
\hline Dasyprocta fuliginosa & 20 & 1 & 7.2 & $1.45 \pm 0.54$ & Distance \\
\hline \multicolumn{6}{|l|}{ Carnivora } \\
\hline Nasua nasua & 20 & 6 & 0.98 & $0.14 \pm 0.24$ & Anchura fija \\
\hline \multicolumn{6}{|l|}{ Edentado } \\
\hline Tamandua tetradactyla & 20 & 1 & 0.5 & $0.09 \pm 0.08$ & Anchura fija \\
\hline \multicolumn{6}{|l|}{ Aves } \\
\hline \multicolumn{6}{|l|}{ Craciformes } \\
\hline Penelope jacquacu & 16 & 4 & 12.09 & $9.42 \pm 1.74$ & Distance \\
\hline
\end{tabular}

Tabla 3. Categorías de abundancias de animales de caza y sus equivalentes en densidad y score obtenidos por transectos y entrevistas. Especies resaltadas son aquellas no coincidentes con ambos métodos.

\begin{tabular}{llllll}
\hline \multirow{2}{*}{ Especie } & \multicolumn{1}{c}{ Referencia } & \multicolumn{2}{c}{ Transectos } & \multicolumn{2}{c}{ Entrevistas } \\
\cline { 2 - 6 } & Alta Densidad & Densidad & Cat.a & Score & Cat.b \\
\hline Lagothrix poeppigii & $10.00-26.32^{1,2,3}$ & 21.05 & $\mathrm{~A}$ & 2.97 & $\mathrm{~A}$ \\
Saimiri sciureus & $5.20-17-25^{1,2,3}$ & 16.60 & $\mathrm{~A}$ & 2.97 & $\mathrm{~A}$ \\
\hline Penelope jacquacu & $5.46-9.02^{2,3,4}$ & 9.42 & $\mathrm{~A}$ & 2.64 & $\mathrm{~A}$ \\
\hline Tayassu pecari & $3.90-14.59^{2,3,5}$ & 6.58 & $\mathrm{~A}$ & 3.00 & $\mathrm{~A}$ \\
\hline Pecari tajacu & $1.52-8.54^{2,3,5}$ & 1.52 & $\mathrm{~A}$ & 2.67 & $\mathrm{~A}$ \\
\hline Dasyprocta fuliginosa & $2.91-30.07^{2,3}$ & 1.45 & $\mathrm{~F}$ & 2.51 & $\mathrm{~A}$ \\
Mazama nemorivaga & $0.43^{3}$ & 0.25 & $\mathrm{~F}$ & 2.04 & $\mathrm{~F}$ \\
Nasua nasua & $15.16^{6}$ & 0.14 & $\mathrm{R}$ & 2.65 & $\mathrm{~A}$ \\
\hline Tapirus terrestris & $0.31-0.8^{2,3}$ & 0.14 & $\mathrm{~F}$ & 2.6 & $\mathrm{~A}$ \\
Mazama americana & $0.70-2.96^{2,3}$ & 0.12 & $\mathrm{~F}$ & 2.26 & $\mathrm{~F}$ \\
Tamandua tetradactyla & $6.6^{6}$ & 0.09 & $\mathrm{R}$ & 1.50 & $\mathrm{R}$ \\
\hline
\end{tabular}

${ }^{1}$ Aquino et al. 2000, ${ }^{2}$ Salovaara et al. 2003, ${ }^{3}$ Peres \& Palacios 2007, ${ }^{4}$ Begazo \& Bodmer 1998, ${ }^{5}$ Aquino et al. 1999,

${ }^{6}$ Robinson \& Redford 1986 . ${ }^{a}$ Categoría basadas en las referencias de densidades bibliográficas,

${ }^{b}$ Categorías creadas de acuerdo al rango, de 1 (raro) a 3 (abundante). A: abundante, F: frecuente, R: raro. 


\section{DISCUSIÓN}

Las especies consideradas como abundantes mediante transectos y entrevistas fueron mayormente aquellas que forman grupos y pueden ser escuchadas a largas distancias como el choro L. poeppigi, fraile S. sciureus, pucacunga P. jacquacu, huangana T. pecari y sajino P. tajacu. Pero también estas especies son abundantes debido a los lineamientos de manejo que regula la caza mediante el establecimiento de cuotas e identificación de especies que soportan la cacería, resultando en una cacería sostenible dentro de la reserva (Pérez-Peña et al., 2013). Entre las especies que no soportan la caza, por lo tanto se prohíbe su cacería al interior de la reserva, esta el mono choro L. poeppigii, que forma grandes grupos, activos en la búsqueda de alimento y tiene una dieta muy diversa en contraste a la maquizapa Ateles belzebuth y el mono coto Alouatta seniculus (Di Fiore, 2003; Aquino \& Bodmer 2004; Dew, 2005) esta característica ayuda a que sea la especie más abundante, pero muy sensible a la sobrecaza (Aquino \& Bodmer, 2001; Peres \& Palacios, 2007). El fraile S. scireus es también abundante y forma grandes grupos pero no es una especie preferida por los cazadores (Bodmer, 1995), y tiene diferentes tácticas frente a la escasez de alimento, consumiendo insectos, flores y exudados (Stone, 2007) y no es impactado negativamente por la cacería (Peres \& Palacios, 2007), todas estas características contribuyen a que sea una de las especies más abundantes.

Entre las especies aptas para la caza, la pucacunga $P$. jacquacu puede soportar la cacería pero desaparece o es rara cuando es excesivo (Begazo \& Bodmer, 1998; Barrio, 2011), la huangana T. pecari puede soportar la caza debido a su estrategia reproductiva, incrementando el número de fetos por hembra en lugares con caza intensa (Fang et al., 2008) aunque otros autores mencionan que su abundancia declina en hábitats con mucha caza (Reyna-Hurtado \& Tanner, 2005; Peres \& Palacios, 2007). Esta especie presenta poblaciones oscilantes en décadas, la cual es muy sensible a la reducción por factores dependientes de la densidad. El sajino P. tajacu tiene similar estrategia reproductiva a la huangana en lugares de caza intensa, elevando su productividad, pero tiene poblaciones menos oscilante y por lo tanto es menos susceptible a la sobrecaza (Pérez-Peña, 2007; Fang et al., 2008).

El venado rojo Mazama americana y el venado gris Mazama nemorivaga también son especies aptas para la caza y fueron considerados como frecuentes; el shihui Tamandua tetradactyla especie no apta para la caza fue considerada como raro usando ambos métodos. El venado rojo puede tener similar densidad en lugares con ninguna, poca o mucha caza
(Peres \& Palacios, 2007), y puede tener mayor productividad anual que el venado gris aunque la sostenibilidad de la caza de ambas especies depende de la presencia de pecaríes o roedores grandes (Hurtado-Gonzales \& Bodmer, 2004). La diferencia en la densidad calculada por transectos, entre ambas especies de venados, puede ser debido a la diferencia en los patrones de actividad, aunque ambos venados son catemerales pero puede ser que el venado gris sea más diurno y el venado rojo más nocturno (Tobler et al., 2009). El shihui fue considerado raro por sus bajas densidades y por sus baja tasa de encuentros por los cazadores kichwas, es catemeral (Aquino et al., 2001) pero puede ser más nocturno que diurno, produce una sola cría y lo cuida durante un año (Medri et al., 2006), es una especie muy lenta y se camufla muy bien entre las ramas del dosel.

Los resultados muestran incongruencias en tres especies, las que fueron consideradas abundantes por los cazadores pero frecuentes o raros por los biólogos que usan transectos lineales o en banda. El añuje Dasyprocta fulignosa fue una de las una especies considerada abundante por los cazadores pero frecuente para los biólogos utilizando transectos lineales. Esta incongruencia puede ser debido a la poca eficiencia del método por transecto lineal en algunas especies. Pereira et al. (2011) evaluaron el uso de trampas cámaras y transectos lineales para el monitoreo de esta especie y concluyó que ambos métodos tienden a fallar en la detección de este roedor. El tamaño poblaciones de especies que viven en madrigueras de suelo o palos caídos, usualmente son subestimadas al emplear transectos lineales (Beck-King et al., 1999), por lo tanto, la percepción de los cazadores en la abundancia de esta especie puede ser más real que los resultados de censos, porque los cazadores además del avistamiento se valen de otras señales como huellas, restos de comida y sonidos

El achuni Nasua nasua fue considerada abundante por los cazadores porque ellos lo ubican por el olor, huellas y rastros que dejan en el suelo, pero fue considerado raro usando transectos. Esta especie al sentir la presencia del censador se inmoviliza, pudiendo ser desapercibida si se localiza detrás de algún árbol frondoso y así resultar en pocos avistamientos. La sachavaca Tapirus terrestris fue considerada abundante por los cazadores pero frecuente en los censos. Esta especie es más activa entre las 18:00 h y las 5:00 h visitando colpas (Tobler et al., 2009); nuestros censos se realizaron entre las 7:00 y 15:00 h motivo por el cual nuestro cálculo de densidad está subestimado. De esta forma se reconoce el amplio conocimiento de los cazadores kichwas en la abundancia de estas especies frente al método por transectos lineales o en banda. 
Sería muy importante conocer la influencia de la probabilidad de detección de las especies en ambos métodos y así determinar cual método es más dependiente de este factor. El presente estudio analizó cuatro especies con el método Distance el cual brinda resultados de probabilidad de detección, sin embargo las demás especies carecen de este valor crucial porque se usó el método de ancho fijo por tener reducido número de avistamientos.

El conocimiento local debe ser tomado en cuenta al momento de decidir las estrategias de monitoreo de la abundancia de animales de caza porque se correlacionan con el conocimiento académico de las especies muy bien muestreadas y tienen mejor conocimiento en aquellas con errores de muestreo. Los indígenas son grandes conocedores de sus animales de caza (Van Holt et al., 2009), esta amalgama de conocimiento debe de ser usado para el beneficio de la propia comunidad local y la conservación de la Amazonía, brindando información a los tomadores de decisiones de forma simple y práctica, y así mostrar que los pobladores indígenas tienen mucho que aportar en el monitoreo y conservación de nuestra vasta amazonia peruana muy estropeada por actividades extractivistas industriales. Este tipo de monitoreo, práctico, sencillo con la exigencia académica permite detectar problemas del mal uso del recurso y puede ayudar al "buen vivir" del poblador indígena amazónico alertando tempranamente en la desaparición de sus animales de caza, principal fuente proteica. Muchas veces los indígenas saben que esta pasando en el bosque "en sus idioma", por lo tanto para informarnos, solo nos queda preguntarlos y escucharlos.

\section{AGRADECIMIENTOS}

Al SERNANP por el apoyo financiero en las actividades censales y entrevistas. A Diana Meza, Ricardo Villacorta y Daniel Montes y guardaparques por su apoyo en la colecta de datos en la reserva y comunidad Kichwa. A Tracy Van Holt por su apoyo en el análisis de consenso cultural y a los cazadores kichwas quienes fueron nuestra motivación.

\section{BIBLIOGRAFÍA CITADA}

Aquino, R.; Bodmer, R. 2004. Plantas útiles en la alimentación de primates en la cuenca del río Samiria, Amazonía peruana. Neotropical Primates, 12(1): 1-6.

Aquino, R.; Bodmer, R.; Gil, G. 2001. Mamiferos de la cuenca del río Samiria: ecología poblacional y sustentabilidad de la caza. Publicación Junglevagt for Amazonas, AIF-WWF/DK WCS. Lima, Perú. 108 pp.
Aquino, R.; Bodmer, R.E.; Pezo, E. 2000. Evaluación de primates en la cuenca del río Pucacuro. La Primatología en el Perú Vol II: 102-110

Aquino, R.; Bodmer, R.E.; Pezo, E. 1999. Evaluación de poblaciones del pecarí de collar (Tayassu tajacu) y pecarí labiado (Tayassu pecari) en la cuenca del río Pucacuro. In: Manejo y conservación de fauna silvestre en América Latina. Fang, T.; Montenegro, O.; Bodmer, R.E. (Eds). Museo de Historia Natural Noel Kempff Mercado, University of Florida, Instituto de ecología, WCS. La Paz, Bolivia. p. 469-478

Ayres, M.; Ayres Jr, M.; Lima-Ayres, D.; Dos Santos, A. 2007. Bioestat. Aplicacioes estatisticas nas áreas das ciencias bio-medicas. Belem, Para, Brasil.

Barrio, J. 2011. Hunting pressure on cracids (Cracidae: Aves) in forest concessions in Peru. Revista Peruana de Biología, 18(2):225-230

Beck-King, H.; Von Helversen, O,; Beck-King, R. 1999. Home range, population density, and food resources of Agouti paca (Rodentia: Agoutidae) in Costa Rica: A study using alternative methods. Biotropica 31(4): 675-685

Begazo, A,J.; Bodmer, R.E. 1998. Use and conservation of Cracidae (Aves: Galliformes) in the Peruvian Amazon. Oryx, 32(4):301-309

Bodmer, R.; Eisenberg, J.; Redford. K. 1997. Hunting and the likelihood of extinction of Amazonian mammals. Conservation Biology. 11 (2). 460-466

Bodmer, R.; Pezo, E.; Fang, T. 2004. Economic analysis of wildlife use in the Peruvian Amazon. In: People in nature. Wildlife conservation in south and central America. Silvius, K.; Bodmer, R.; Fragoso, J. (Eds). Columbia University Press, New York. P. 191-207

Bodmer, R.E.; Puertas, P.; Antúnez, M.; Fang, T.; Pérez-Peña, P.E.. 2011. Monitoreo de Especies indicadoras para evaluar el impacto del cambio climático en la cuenca del Samiria, Reserva Nacional Pacaya Samiria. Documento Técnico. W C S , O P W A L L, S E R N A N P, FUNDAMAZONIA, DICE, , EARTHWATCH. $207 \mathrm{pp}$

Bodmer, R.E. 1995. Managing amazonian wildlife: Biological correlates of game choice by detribalized hunters. Ecological Applications, 5(4):872-877

Borgatti, S.P.; Everett, M.G.; Freeman, L.C. 2002. Ucinet for Windows: Software for social network analysis. Harvard, MA: Analytic Technologie. 
Buckland, S.T.; Anderson, D.; Burnham, K.; Laake. J. 1993. Distance sampling: Estimating the abundance of biological populations. Chapman \& Hall, London. $441 \mathrm{pp}$.

Burnham, K.P; Anderson, D.R.; Laake, J. 1980. Estimation of density from line transect sampling of biological populations. Wildlife Monographs, 72: 3-202

Dew, J.L. 2005. Foraging, food choice, and food processing by sympatric ripe-fruit specialists: Lagothrix lagotricha poeppigii and Ateles belzebuth belzebuth. International Journal of Primatology, 26(5):1107-1135

Di Fiore, A. 2004.Diet and feeding ecology of woolly monkeys in a western Amazonian rain forest. International Journal of Primatology, 25(4):767-801

Eisenberg, J.; Redford, K. 1999. Mammals of the neotropics. The central neotropics: Volumen 3. Ecuador, Perú, Bolivia, Brazil. The University of Chicago Press. 606 pp.

Elzinga, C.L.; Salzer, D.W.; Willoughby, J.W.; Gibbs, J. 2001. Monitoring plant and animal populations. Blackwell Science. Reino Unido. $360 \mathrm{pp}$.

Emmons, L. H. 1997. Neotropical Rainforest Mammals: A Field Guide. 2da. Ed. The University of Chicago Press, Chicago, IL.

Fang, T.; Bodmer, R.E.; Puertas, P.; Mayor, P.; Pérez-Peña, P.E.; Acero, R.; Haymann, D. 2008. Certificación de pieles de pecaríes en la Amazonía peruana: Una estrategia para la Conservación y Manejo de Fauna en la Amazonía Peruana. Wust Ediciones. Lima, Perú. 203 pp

Fragoso, J.M.V. 2004. A long - term study of whitelipped peccary (Tayassu pecari) population fluctuation in northern Amazonia. Anthropogenic vs "natural" causes. In: People in Nature. Wildlife conservation in south and central America. Silvius, K.; Bodmer, R.; Fragoso, J.M.V. (Eds). Columbia University Press. New York. p. 286-296.

Haugaasen, T.; Peres, C. A.; 2007. Vertebrate responses to fruit production in Amazonian flooded and unflooded forests. Biodiversity Conservation. 16: 4165-4190.

Hurtado-Gonzales, J.; Bodmer. R. 2004. Assessing the sustainability of brocket deer hunting in the Tamshiyacu Tahuayo Communal Reserve, northeastern Peru. Biological Conservation. 116. 1-7.

Medri, I.M.; Mourao, G.; Rodrigues, F.H. 2006. Ordem Xenarthra. In: Mamiferos do Brasil. Reis, N.; Peracchi, A.L.; Lima, W.P.; Lima, I.P. (Eds).Londrina, Parana, Brasil. p. 71-99
Meredith, M. 2008. Estimating population size with line transect and DISTANCE. In: ProblemSolving in Conservation Biology and Wildlife Management. Gibbs, J.; Hunter, H.; Sterling, E. (Eds). Blackwell Publishing. 2a Ed. p. 88-104

Pereira-Munaria, P.; Kellerb, C.; Venticinquec, E. 2011. An evaluation of field techniques for monitoring terrestrial mammal populations in Amazonia. Mammalian Biology. 76 (4): 401-408.

Peres, C. A.; Cunha, A.A. 2011. Manual para censo e monitoramento de vertebrados de médio e grande porte por transecção linear em florestas tropicais. Wildlife Technical Series, Wildife Conservation Society, Brasil. $25 \mathrm{pp}$

Peres, C.; Palacios, E. 2007. Basin-wide effects of game harvest on vertebrate population densities in Amazonian forest: Implications for animalmediated seed dispersal. Biotropica, 39(3):304315

Peres, C.; Dolman, P. 2000. Density compensation in neotropical primate communities: evidence from 56 hunted and nonhunted Amazonian forests of varying productivity. Oecologia, 122:175-189

Perez-Peña, P.E.; Ruck, L.; G. Rojas. 2013. Evaluación de la caza de la etnia kichwa en la zona de caza de la Reserva Nacional Pucacuro. Revista Virtual FaunaVET-Perú. www.faunavet-peru.com

Pérez-Peña, P.E. 2007. Refining the guidlines of the peccary pelt certification programme. Tesis de Maestría, Durrell Institute of Conservation and Ecology, Universidad de Kent, Canterbury, Reino Unido. $75 \mathrm{pp}$.

Pitman, N.; Pinedo-Cecilio, M.; Pinedo-Pudicho, M.; Graham, J.; Nuñez, P.; Valenzuela, M.; Terborgh, J. 2011. Indigenous perceptions of tree species abundance across an upper Amazonian landscape. Journal of Ethnobiology. 31(2):233-243

Reis, N.; Peracchi, A.L.; Lima, W.P.; Lima, I.P. 2006. Mamíferos do Brasil. Londrina, Parana, Brasil. $437 \mathrm{pp}$.

Reyna-Hurtado, R.; Tanner, G.W. 2005. Habitat Preferences of Ungulates in Hunted and Nonhunted Areas in the Calakmul Forest, Campeche, Mexico. Biotropica. 37(4):676-685

Robinson, J.; Redford, K. 1986. Body Size, diet, and population density of neotropical forest mammals. The American Naturalist. 128(5):665-680

Rowcliffe, M. 2002. Bushmeat and the biology of conservation. Oryx. 36(4): 331.

Salovaara, K.; Bodmer, R.; Recharte, M.; Reyes, C. 2003. Diversidad y Abundancia de Mamíferos. 
In: Perú: Yavarí. Rapid Biological Inventories. The Field Museum. Report 11.p. 74-84.

Schulenberg, T.; Stotz, D.; Lane, D.; O’Neill, J.; Parker III, T.. 2010. Aves de Perú. Serie en Biodiversidad Corbidi 01. CORBIDI. Lima, Perú. 460 pp.

Stone, A.I. 2007. Responses of squirrel monkeys to seasonal changes in food availability in an eastern Amazonian forest. American Journal of Primatology, 69:142-157

Terborgh, J.; Robinson, S.; Parker, T.; Munn, C.; Pierpoint, N. Structure and Organization of an Amazonian forest bird Community. Ecological Monographs, 60(2): 213-238

Thomas, L.; Laake, J.L.; Rexstad, E.; Strindberg, S.; Marques, F.F.C.; Buckland, S.T.; Borchers, D.L.; Anderson, D.R.; Burnham, K.P.; Burt, M.L.; Hedley, S.L.; Pollard, J.H.; Bishop, J.R.B.; Marques, T.A. 2009. Distance 6.0. Release "2". Research Unit for Wildlife
Population Assessment, University of St. Andrews, UK.

Tobler, M.T.; Carrillo-Percastegui, S.E.; Powell, G. 2009. Habitat use, activity patterns and use of mineral licks by five species of ungulate in southeastern Peru. Journal of Tropical Ecology, 25:261-270

Van Holt, T.; Towsend, W. R.; Cronkleton, P. 2010. Assessing local knowledge of game abundance and persistence of hunting livelihoods in the Bolivian Amazon using consensus analysis. Human Ecology. 38: 791-801

Voss, R.; Fleck, D. 2011. Mammalian diversity and Matses ethnomammalogy in Amazonian Peru. Part 1: Primates. Bulletin of the American Museum of Natural History. 351:1-81

Weller, S. C. 2007. Cultural consensus theory: applications and frequently asked questions. Field Methods, 19:339-368. 